\title{
A robust numerical method to study oscillatory instability of gap solitary waves
}

\author{
Gianne Derks and Georg A. Gottwald \\ Dept. of Mathematics and Statistics, University of Surrey, Guildford, Surrey, GU2 7XH, UK \\ School of Mathematics and Statistics, University of Sydney, NSW 2006, Australia
}

October 1, 2018

\begin{abstract}
The spectral problem associated with the linearization about solitary waves of spinor systems or optical coupled mode equations supporting gap solitons is formulated in terms of the Evans function, a complex analytic function whose zeros correspond to eigenvalues. These problems may exhibit oscillatory instabilities where eigenvalues detach from the edges of the continuous spectrum, so called edge bifurcations. A numerical framework, based on a fast robust shooting algorithm using exterior algebra is described. The complete algorithm is robust in the sense that it does not produce spurious unstable eigenvalues. The algorithm allows to locate exactly where the unstable discrete eigenvalues detach from the continuous spectrum. Moreover, the algorithm allows for stable shooting along multi-dimensional stable and unstable manifolds. The method is illustrated by computing the stability and instability of gap solitary waves of a coupled mode model.
\end{abstract}

Key words: gap solitary wave, numerical Evans function, edge bifurcation, exterior algebra, oscillatory instability, massive Thirring model

AMS subject classifications: 65P30, 65P40, 37M20, 74J35 


\section{Introduction}

Transmitting information efficiently across long optical waveguides is a big challenge in telecommunications. Gap solitons are potential candidates to achieve this goal. A gap in the linear spectrum allows solitons in spinor-like systems to propagate without losing energy due to a resonant interaction with linear waves 11, 18. For example, an optical fiber with a periodically varying refractive index supports gap solitons. The gap here is created by Bragg reflection and resonance of waves along the grating.

Before its application to optical waveguides and transmission of optical pulses, gap solitons have been studied in the context of spinor field equations in elementary particle physics [19, 20] and in condensed matter physics [9]. There is an extensive literature on the existence of gap solitons, but the issue of their stability, which is of paramount practical importance, is still an open question for many systems.

Gap solitons were long believed to be stable. This was conjectured on the grounds of computer simulations [1] in (restricted) parameter regimes. Only recently BARASHENKOV, PELINOVSKY \& ZEMLYANAYA [4] showed analytically by using a perturbation theory, and BARASHENKOV \& ZEMLYANAYA [5] verified numerically that, in a particular optical system, gap solitons can undergo oscillatory instabilities where eigenvalues detach from the edges of the continuous spectrum (edge bifurcations). In Kapitula \& SAndstede [15, Evans functions are used to detect analytically the onset of an oscillatory instability (edge bifurcation) in near integrable systems. From an analytical point of view a difficulty in proving stability/instability is that the energy in these systems is neither bounded from above nor from below. Usual techniques such as energy-momentum methods or methods involving Lyapunov functionals are therefore bound to fail.

An often used numerical approach to eigenvalue problems is to discretize the spectral problem on the truncated domain $x \in\left[-L_{\infty}, L_{\infty}\right]$ (with $L_{\infty} \gg 1$ ) using finite differences, collocation or spectral methods, reducing it to a very large matrix eigenvalue problem. There are two central difficulties with this approach. Firstly, in general the exact asymptotic boundary conditions at $x= \pm L_{\infty}$ depend on the eigenvalue $\lambda$ in a nonlinear way, and so application of the exact asymptotic boundary conditions changes the problem to a matrix eigenvalue problem, which is nonlinear in the parameter. So matrix eigenvalue solvers can no longer be used. In the above papers 4, 5], artificial boundary conditions such as Dirichlet or periodic boundary conditions, were applied, in order to retain linearity in the spectral parameter. For a detailed discussion on artificial boundary conditions, see SAndstede \& Scheel [23, 24]. Secondly, the approximate boundary conditions lead often to spurious discrete eigenvalues generated from the fractured continuous spectrum. If the continuous spectrum is strongly stable (that is, the continuous spectrum is stable and there is a gap between the continuous spectrum and the imaginary axis) this does not normally generate spurious unstable eigenvalues. However, if the continuous spectrum lies on the imaginary axis (as often happens with gap solitons due to the Hamiltonian character of the underlying system), spurious eigenvalues may be emitted into the unstable half plane.

Indeed, BARASHENKov \& ZEMLYANAyA [5] give an extreme example, where a large number of spurious unstable eigenvalues are generated by the matrix discretization (see Figure 1 in [5]). These problems prohibit their method to be used as a robust scheme to check stability when no theoretical results are given to guide the experiments.

A robust and stable numerical scheme is still an open problem. In this paper we present a numerical method based on the Evans function and exterior algebra which does not exhibit spurious unstable eigenvalues. The Evans function is a complex analytic function whose zeros correspond to eigenvalues of the spectral problem associated with the linearization about a solitary wave solution. The Evans function was first introduced by Evans [12] and generalized by AlEXANDER, Gardner \& Jones [2]. To define the Evans function, one writes the wave equations as a system of first order real equations with respect to the spatial variable $x$, such that one gets a system 
of the form $\mathbf{Z}_{x}=F\left(\mathbf{Z}, \mathbf{Z}_{t}, \mathbf{Z}_{t t}, \ldots\right)$. The study of linear stability of a solitary wave solution $\widehat{\mathbf{Z}}(x)$ involves a linearisation about $\widehat{\mathbf{Z}}$ by writing the basic solutions as $\mathbf{Z}(x)=\widehat{\mathbf{Z}}(x)+\mathbf{u}(x) e^{\lambda t}$. This will lead to a linearized problem of the form

$$
\mathbf{u}_{x}=\mathbf{A}(x, \lambda) \mathbf{u}, \quad \mathbf{u} \in \mathbb{C}^{n},
$$

where $\lambda \in \mathbb{C}$ is the spectral parameter and $\mathbf{A}(x, \lambda)$ is a matrix in $\mathbb{C}^{n \times n}$, whose limit for $x \rightarrow \pm \infty$ exists. The solitary wave solution $\widehat{\mathbf{Z}}$ of a partial differential equation is linearly unstable if for a spectral parameter $\lambda$ with $\Re(\lambda)>0$ there exists an associated perturbation $\mathbf{u}(x)$, which is bounded for all $x$. An oscillatory instability (edge bifurcation) does happen when the continuous spectrum is on the imaginary axis and one of the bounded eigenfunctions of the continuous spectrum develops into an exponentially decaying eigenfunction with $\Re(\lambda)>0$.

To verify if such bounded eigenfunction $\mathbf{u}(x)$ exists for a given value of $\lambda$ with $\Re(\lambda)>0$, it is checked if the unstable manifold at $x=-\infty$ and the stable manifolds at $x=\infty$ have a non-trivial intersection. If this is the case, the solitary wave solution $\widehat{\mathbf{Z}}$ is unstable with positive growth rate $\Re(\lambda)>0$. To check the transversality condition the Evans function is used. Hence the Evans function can be viewed as a Melnikov function or a Wronskian determinant.

Crucial to the initial construction of the Evans function is the distribution of the eigenvalues of the 'system at infinity', that is, the matrix $\mathbf{A}_{ \pm \infty}(\lambda)$ which is associated with the limit as $x \rightarrow \pm \infty$ of $\mathbf{A}(x, \lambda)$. It is assumed that no eigenvalues are on the imaginary axis ${ }^{1}$ and that the number of negative eigenvalues is constant for $\lambda \in \Lambda$, where $\Lambda$ is a simply-connected subset of $\mathbb{C}$. Let $k$ be the number of negative eigenvalues of $\mathbf{A}_{ \pm \infty}(\lambda)$ for $\lambda \in \Lambda$. Note that in the case of different asymptotic behaviour at $x= \pm \infty$ as for example in the case of fronts, $k$ can be different at $x= \pm \infty$ in general.

This suggests a naive approach by which one may follow the stable/unstable manifolds at $x= \pm \infty$ with a standard shooting method and check their intersection using the Evans function. This may be done indeed if the dimension of these manifolds is 1. Otherwise, any integration scheme will inevitably just be attracted by the eigendirection corresponding to the most unstable eigenvalue. However, for most systems, the dimensional of the stable/unstable manifold will be larger than 1. In this paper, we will consider a model equation with stable/unstable manifolds of dimension 2. To keep the eigendirections orthogonal in the course of the numerical integration, one may employ a Gram-Schmidt orthogonalization method. However, this is a non-analytic procedure, which will eventually backfire in the case of oscillatory instabilities where we expect zero's of the Evans function in the complex plane. Indeed, to locate those complex eigenvalues, Cauchy's Theorem (argument principle) will be employed and thus an analytical method is crucial. In our numerical method, we will use exterior algebra, which allows for an analytical calculation of the Evans function.

The numerical method builds on the work in 3, 10, where a numerical algorithm is given for the calculation of the Evans function and applications are give to the stability and instability in a fifth-order $\mathrm{KdV}$ equation. In this paper, we will show that a similar algorithm can be used to determine oscillatory instabilities (edge bifurcations). The algorithm does not exhibit spurious eigenvalues and the exact asymptotic boundary conditions are built into the definition of the Evans function in an analytic way. The analyticity can then be utilized to apply Cauchy's principle value theorem to study stability/instability. An important feature of the numerical method is that it involves the use of exterior algebra to describe the system on a higher dimensional space in which a simple shooting method can be employed. A similar idea is used in BRIN [7] and BRIN \& ZuMBrun [8] for dealing with instabilities in viscous fluid flows.

\footnotetext{
${ }^{1}$ The assumption on the hyperbolicity of $\mathbf{A}_{ \pm \infty}(\lambda)$ can be weakened (see [13 14]).
} 
To illustrate our method we will consider the following coupled mode model

$$
\begin{aligned}
& 0=\mathrm{i}\left(u_{t}+u_{x}\right)+v+\left(|v|^{2}+\rho|u|^{2}\right) u \\
& 0=\mathrm{i}\left(v_{t}-v_{x}\right)+u+\left(|u|^{2}+\rho|v|^{2}\right) v .
\end{aligned}
$$

This is a model to describe optical pulses in waveguides which have been grated so the refractive index is varying periodically. In the case $\rho=0$ this equation is known in field-theory as the massive Thirring model and was shown to be completely integrable (see for example [1, 16]). In a nonlinear optics context, one has $\rho=1 / 2$ in periodic Kerr media [11, but in other media $\rho$ may range from 0 up to infinity [22]. The equation (1.2) has also been studied by BARASHENKOV, Pelinovsky \& Zemlyanaya [4], Barashenkov \& Zemlyanaya 5] and in a slightly modified form by KAPITUla \& SANDSTEDE [15]. In [4, 5] a heuristic perturbation analysis is used to analyse the onset of oscillatory instabilities and numerical study is used to give a more complete picture. The numerical study encountered serious problems as discussed above. In [15], a relation between the Evans function and the inverse scattering formalism is established for integrable systems. This forms the basis of a rigorous perturbation analysis for perturbations of the massive Thirring model.

The coupled mode model (1.2) is chosen for illustration purposes, since the work in the previous papers allows us to illustrate the advantages of our method. We stress though that the method we present is general and can be applied to gap solitons in other systems as well. Moreover, there is no need for the system to be related to an integrable system.

\section{The numerical Evans function for the coupled mode mode}

The solutions and dynamics of (1.2) are best described by splitting off the real and imaginary part of the fields $u, v$. We shall write for a solution $u=Q_{1}+i P_{1}$ and $v=Q_{2}+i P_{2}$ and collect the information in a single real solution vector $\mathbf{Z}=\left(Q_{1}, Q_{2}, P_{1}, P_{2}\right)$.

\subsection{The model equations and its solutions}

We introduce the Lorentz transformation $X=(x-V t) / \sqrt{1-V^{2}}$ and $T=(t-V x) / \sqrt{1-V^{2}}$. In these boosted variables the model system (1.2) may be written in a (quasi-)multisymplectic framework as

$$
\mathbb{E}\left[\mathbb{M} \mathbf{Z}_{T}+\mathbb{K} \mathbf{Z}_{X}\right]=\nabla S(\mathbf{Z})
$$

where $\mathbf{Z}=\left(Q_{1}, Q_{2}, P_{1}, P_{2}\right)$,

$$
\begin{aligned}
& S(Z)=\frac{1}{2}\left[Q_{1} Q_{2}+P_{1} P_{2}+\left(Q_{1}^{2}+P_{1}^{2}\right)\left(Q_{2}^{2}+P_{2}^{2}\right)\right]+\frac{\rho}{2}\left[\left(Q_{1}^{2}+P_{1}^{2}\right)^{2}+\left(Q_{2}^{2}+P_{2}^{2}\right)^{2}\right], \\
& \mathbb{E}=\left(\begin{array}{cc}
\mathbb{E}_{1} & 0 \\
0 & \mathbb{E}_{1}
\end{array}\right), \quad \mathbb{E}_{1}=\left(\begin{array}{cc}
e^{-y} & 0 \\
0 & e^{y}
\end{array}\right), \quad \mathbb{M}=\left(\begin{array}{cc}
0 & \sigma_{0} \\
-\sigma_{0} & 0
\end{array}\right) \quad \text { and } \quad \mathbb{K}=\left(\begin{array}{cc}
0 & \sigma_{3} \\
-\sigma_{3} & 0
\end{array}\right),
\end{aligned}
$$

with $V=\tanh y$ and the Pauli matrices $\sigma_{0,3}$ are defined as

$$
\sigma_{0}=\left(\begin{array}{ll}
1 & 0 \\
0 & 1
\end{array}\right) \quad \text { and } \quad \sigma_{3}=\left(\begin{array}{cc}
1 & 0 \\
0 & -1
\end{array}\right)
$$

Note that if $\rho=0$, the system is invariant under this Lorentz transformation. 
The reason for introducing this formalism is that a multisymplectic formulation allows for a systematic linearization and also sheds light on conservation properties within the system. We note that (2.1) is equivariant under action of the continuous symmetry group $S O(2)$ acting on $\mathbb{R}^{4}$ represented by

$$
G_{\psi}=\left(\begin{array}{cc}
\cos (\psi) \sigma_{0} & -\sin (\psi) \sigma_{0} \\
\sin (\psi) \sigma_{0} & \cos (\psi) \sigma_{0}
\end{array}\right),
$$

since $\left[G_{\psi}, \mathbb{M}\right]=\left[G_{\psi}, \mathbb{K}\right]=\left[G_{\psi}, \mathbb{E}\right]=0$ and $S\left(G_{\psi} \mathbf{Z}\right)=S(\mathbf{Z})$ for any $\psi$. According to Noether's Theorem, there is a conservation law associated with this continuous symmetry, namely $P_{T}+$ $Q_{X}=0$ with $P$ and $Q$ determined by

$$
\nabla P(\mathbf{Z})=\left.\mathbb{M} \frac{d}{d \psi}\right|_{\psi=0} G_{\psi}(\mathbf{Z}) \quad \text { and } \quad \nabla Q(\mathbf{Z})=\left.\mathbb{K} \frac{d}{d \psi}\right|_{\psi=0} G_{\psi}(\mathbf{Z})
$$

hence

$$
P(\mathbf{Z})=\sum_{i=1}^{2} Q_{i}^{2}+P_{i}^{2} \quad \text { and } \quad Q(\mathbf{Z})=\sum_{i=1}^{2}(-1)^{i+1}\left(Q_{i}^{2}+P_{i}^{2}\right) .
$$

Note that in the original system (1.2), this symmetry shows up as an equivariance of the system under simultaneous phaseshifts of $u$ and $v$, i.e., $u \mapsto u e^{\mathrm{i} \psi}$ and $v \mapsto v e^{\mathrm{i} \psi}$.

Going to a frame moving with the symmetry group and writing the solutions as $\mathbf{Z}(X, T)=$ $G_{\varphi(X)-\Omega T} \widetilde{\mathbf{Z}}(X, T)$, the equations (2.1) become

$$
\mathbb{E}\left[\mathbb{M} \mathbf{Z}_{T}+\mathbb{K} \mathbf{Z}_{X}-\Omega \nabla P(\mathbf{Z})+\varphi_{X} \nabla Q(\mathbf{Z})\right]=\nabla S(\mathbf{Z})
$$

where we dropped the tildes. Time independent solutions in the moving frame were found by Aceves \& Wabnitz [1] to be

$$
\hat{\mathbf{Z}}(X)=\alpha \mathbb{E}^{-\frac{1}{2}}\left(\begin{array}{cc}
\sigma_{3} & 0 \\
0 & \sigma_{0}
\end{array}\right)\left(\begin{array}{c}
W_{r}(X) \\
W_{r}(X) \\
W_{i}(X) \\
W_{i}(X)
\end{array}\right),
$$

where

$$
\begin{aligned}
& \Omega=\cos \theta(0<\theta<\pi), \quad \varphi(X)=2 \alpha^{2} \rho \sinh (2 y) \arctan \left\{\tanh [(\sin (\theta)) X] \tan \frac{\theta}{2}\right\}, \\
& \alpha=\frac{1}{\sqrt{1+\rho \cosh 2 y}} \text { and } W(X)=W_{r}(X)+\mathrm{i} W_{i}(X)=\frac{\sin (\theta)}{\cosh ((\sin \theta) X-\mathrm{i} \theta / 2)} .
\end{aligned}
$$

Note that there cannot be any gap solitons for $|\Omega|>1$ as then there is no gap in the linear spectrum. At the upper edge of the gap, at $\theta \rightarrow 0(\Omega \rightarrow 1)$, the solutions approaches the smallamplitude nonlinear Schrödinger soliton $W(X)=\theta \operatorname{sech}(\theta(X-\mathrm{i} / 2))$. At the lower edge, at $\theta \rightarrow \pi$ $(\Omega \rightarrow-1)$, the gap soliton has a finite amplitude and decays algebraically, $W(X)=\mathrm{i} /(X+\mathrm{i} / 2)$. These two limits are referred to as "low intensity" and "high intensity" limits, respectively [11. 


\subsection{The linearized problem}

To study the stability of the solution (2.5), we linearize around this solution in the moving frame and use a spectral Ansatz

$$
\mathbf{Z}(X, T)=\left(\widehat{\mathbf{Z}}(X)+\mathbf{u}(X) e^{\lambda T}\right) .
$$

We obtain the dynamical system

$$
\mathbf{u}_{X}=\mathbf{A}(X, \lambda) \mathbf{u}, \quad \mathbf{u} \in \mathbb{C}^{4}
$$

with

$$
\mathbf{A}(X, \lambda)=\mathbb{K}^{-1}\left[\mathbb{E}^{-1} D^{2} S(\hat{\mathbf{Z}})+\Omega D^{2} P(\hat{\mathbf{Z}})-\varphi_{X} D^{2} Q(\hat{\mathbf{Z}})-\lambda \mathbb{M}\right]
$$

where $D^{2}$ denotes the Hessian.

\subsection{Asymptotic properties of the linearised system}

As described in the Introduction, the eigenvalues and eigenfunctions of the asymptotic matrix $\mathbf{A}_{\infty}(\lambda)=\lim _{x \rightarrow \pm \infty} \mathbf{A}(x, \lambda)$ are crucial for the numerical Evans function approach. The matrix $\mathbf{A}(x, \lambda)$ has the asymptotic property that

$$
\mathbf{A}_{\infty}(\lambda)=\lim _{x \rightarrow \pm \infty} \mathbf{A}(x, \lambda)=\left(\begin{array}{cccc}
-\lambda & 0 & -\Omega & -e^{y} \\
0 & \lambda & e^{-y} & \Omega \\
\Omega & e^{y} & -\lambda & 0 \\
-e^{-y} & -\Omega & 0 & \lambda
\end{array}\right) .
$$

The characteristic polynomial of $\mathbf{A}_{\infty}(\lambda)$ is

$$
\Delta(\mu, \lambda)=\operatorname{det}\left[\mu \mathbf{I}-\mathbf{A}_{\infty}(\lambda)\right]=\left(\mu^{2}-\lambda^{2}-1+\Omega^{2}\right)^{2}+4 \Omega^{2} \lambda^{2} .
$$

Thus the eigenvalues $\mu$ of the asymptotic matrix satisfy

$$
\mu^{2}-\lambda^{2}-1+\Omega^{2}= \pm 2 \mathrm{i} \Omega \lambda \quad \text { or } \quad \mu^{2}=1+(\lambda \pm \mathrm{i} \Omega)^{2} .
$$

The continuous spectrum is found by setting $\mathcal{R}(\mu)=0$ or $\mu=\mathrm{i} k$. A short calculation gives that there are four branches of continuous spectrum on the imaginary $\lambda$-axis:

$$
\lambda_{ \pm}(k)=\mathrm{i}\left(\sqrt{1+k^{2}} \pm|\Omega|\right) \quad \text { and } \quad-\lambda_{ \pm}(k), \quad k \in \mathbb{R}
$$

The end points of the continuous spectrum are at $\pm \mathrm{i}(1-|\Omega|)$ and $\pm \mathrm{i}(1+|\Omega|)$. For all values of $\lambda$ not in the continuous spectrum, there are 2 eigenvalues $\mu$ with positive real part and 2 eigenvalues $\mu$ with negative real part. Thus outside the continuous spectrum, there is a $2: 2$ splitting, that is the dimension of the stable/unstable manifolds is 2 at both limits of $x= \pm \infty$.

With (2.10), it is easy to determine explicit expressions for the eigenvalues and eigenvectors. The eigenvalues are

$$
\begin{aligned}
& \mu_{m}^{ \pm}(\lambda)= \pm \sqrt{(\lambda-\mathrm{i}|\Omega|)^{2}+1}= \pm \sqrt{\lambda-\mathrm{i}(|\Omega|+1)} \sqrt{\lambda-\mathrm{i}(|\Omega|-1)} \\
& \mu_{p}^{ \pm}(\lambda)= \pm \sqrt{(\lambda+\mathrm{i}|\Omega|)^{2}+1}= \pm \sqrt{\lambda+\mathrm{i}(|\Omega|+1)} \sqrt{\lambda+\mathrm{i}(|\Omega|-1)}
\end{aligned}
$$


where the square roots are defined as follows:

$$
\sqrt{z}=\sqrt{|z|} \mathrm{e}^{\mathrm{i} \arg (z) / 2}, \quad \text { where } \quad-\pi<\arg (z) \leq \pi \quad \text { and } \quad z=\lambda \pm \mathrm{i}(|\Omega| \pm 1) .
$$

Hence the cuts in the complex plane, associated with this definition of the square roots, start at the end points of the continuous spectrum and continue in the left half of the complex $\lambda$-plane. Note that this definition implies that all square roots have positive real parts.

In this model system it is easy to find explicit expressions for the eigenvectors. The eigenvector with the eigenvalue $\mu_{i}^{ \pm}$for the matrix $\mathbf{A}_{\infty}(\lambda)$ is given by

$$
\begin{aligned}
& \operatorname{vec}_{m}^{ \pm}(\lambda)=\left(F_{m}^{ \pm}, \operatorname{sgn}(\Omega) \mathrm{i} e^{-y},-\operatorname{sgn}(\Omega) \mathrm{i} F_{m}^{ \pm}, e^{-y}\right)^{T}, \quad \text { where } \quad F_{m}^{ \pm}=\lambda-\mu_{m}^{ \pm}-\mathrm{i}|\Omega|, \\
& \operatorname{vec}_{p}^{ \pm}(\lambda)=\left(F_{p}^{ \pm},-\operatorname{sgn}(\Omega) \mathrm{i} e^{-y}, \operatorname{sgn}(\Omega) \mathrm{i} F_{p}^{ \pm}, e^{-y}\right)^{T}, \quad \text { where } \quad F_{p}^{ \pm}=\lambda-\mu_{p}^{ \pm}+\mathrm{i}|\Omega| .
\end{aligned}
$$

The eigenvector with the eigenvalue $\overline{\mu_{i}^{\ddagger}}$ for the adjoint matrix $\left(\overline{\mathbf{A}_{\infty}(\lambda)}\right)^{T}$ is given by

$$
\begin{aligned}
& \operatorname{advec}_{m}^{ \pm}(\lambda)=\left(\overline{F_{m}^{ \pm}}, \operatorname{sgn}(\Omega) \mathrm{i} e^{y},-\operatorname{sgn}(\Omega) \overline{\mathrm{i}} \overline{F_{m}^{ \pm}}, e^{y}\right)^{T}, \quad \text { where } \quad F_{m}^{ \pm}=\lambda-\mu_{m}^{ \pm}-\mathrm{i}|\Omega|, \\
& \operatorname{advec}_{p}^{ \pm}(\lambda)=\left(\overline{F_{p}^{ \pm}},-\operatorname{sgn}(\Omega) \mathrm{i} e^{y}, \operatorname{sgn}(\Omega) \overline{\mathrm{i}} \overline{F_{p}^{ \pm}}, e^{y}\right)^{T}, \quad \text { where } \quad F_{p}^{ \pm}=\lambda-\mu_{p}^{ \pm}+\mathrm{i}|\Omega| .
\end{aligned}
$$

One might also use a numerical routine to solve the eigenvalue problem of $\mathbf{A}_{\infty}(\lambda)$. However, one has to be very careful near the end points of the continuous spectrum. At these points two eigenvalues collide, hence it will be tricky to find numerically the 'correct' eigenvalue. If we fail to do so, this will result in a change of orientation in the Evans function (2.14). This causes unwanted zero's of the Evans function and is reminiscent of the unstable spurious eigenvalues observed when the system (2.6) is solved by some form of discretization as done by BARASHENKOV \& ZEMLYANAYA [5]. One can construct a numerical routine to follow the correct eigenfunction, but if the analytical expressions are readily available (as they are here) it is much easier.

\subsection{The Evans function and the formulation in the exterior algebra}

For $\Re(\lambda)>0$, the system (2.6) and the properties of the system at infinity, $\mathbf{A}_{\infty}(\lambda)$, are in standard form for the dynamical systems formulation of the spectral problem proposed by EvANs 12 . and generalized by Alexander, Gardner \& Jones [2]. A value of $\lambda \in \Lambda$ is an eigenvalue if the 2 -dimensional space of solutions which decays as $x \rightarrow-\infty$ and the the $(4-2)$-dimensional space of solutions which do not grow exponentially as $x \rightarrow+\infty$ have a nontrivial intersection. The Evans function is an analytic function which gives a zero if such a nontrivial intersection exists. To obtain an analytic description of the 2-dimensional space of solutions of (2.6) which do not grow exponentially as $x \rightarrow+\infty$, we will use that the system (2.6) induces a dynamical system on the wedge-space $\bigwedge^{2}\left(\mathbb{C}^{4}\right)$. This is a space of dimension $\left(\begin{array}{l}4 \\ 2\end{array}\right)=6$. To define the Evans function, the induced dynamics on this wedge-space $\bigwedge^{2}\left(\mathbb{C}^{4}\right)$ will be used.

The induced system can be written as

$$
\mathbf{U}_{x}=\mathbf{A}^{(2)}(x) \mathbf{U}, \quad \mathbf{U} \in \bigwedge^{2}\left(\mathbb{C}^{4}\right) .
$$

Here the linear operator (matrix) $\mathbf{A}^{(2)}$ is defined on a decomposable 2 -form $\mathbf{u}_{1} \wedge \mathbf{u}_{2}, \mathbf{u}_{i} \in \mathbb{C}^{4}$, as

$$
\mathbf{A}^{(2)}\left(\mathbf{u}_{1} \wedge \mathbf{u}_{2}\right):=\left(\mathbf{A} \mathbf{u}_{1}\right) \wedge \mathbf{u}_{2}+\mathbf{u}_{1} \wedge\left(\mathbf{A} \mathbf{u}_{2}\right)
$$

and it extends by linearity to the non-decomposable elements in $\bigwedge^{2}\left(\mathbb{C}^{4}\right)$. This construction can be carried out in a coordinate free way and can be generalised to $(k):(n-k)$ splittings in $n$ dimensional dynamical systems. General aspects of the numerical implementation of this theory can be found in Allen \& Bridges [3]. 
Since the induced matrix $\mathbf{A}^{(2)}(x, \lambda)$ inherits the differentiability and analyticity of $\mathbf{A}(x, \lambda)$, the limiting matrices will exist,

$$
\mathbf{A}_{\infty}^{(2)}(\lambda)=\lim _{x \rightarrow \pm \infty} \mathbf{A}^{(2)}(x, \lambda)
$$

The set of eigenvalues of the matrix $\mathbf{A}_{\infty}^{(2)}(\lambda)$ consists of all possible sums of 2 eigenvalues of $\mathbf{A}_{\infty}(\lambda)$ (this is an exercise in multi-linear algebra, see MARcus [21]). Therefore, for $\Re(\lambda)>0$, there is an eigenvalue of $\mathbf{A}_{\infty}^{(2)}(\lambda)$, denoted by $\sigma_{+}(\lambda)$, which is the sum of the 2 eigenvalues of $\mathbf{A}_{\infty}(\lambda)$ with negative real part, i.e., $\sigma_{+}(\lambda)=-\left(\mu_{m}^{+}(\lambda)+\mu_{p}^{+}(\lambda)\right.$ ) (note that the subscript " + " in $\sigma_{+}(\lambda)$ refers to exponentially decaying behaviour at $+\infty)$. Moreover this eigenvalue is simple, an analytic function of $\lambda$ and has real part strictly less than any other eigenvalue of $\mathbf{A}_{\infty}^{(k)}(\lambda)$. Similarly, there is an eigenvalue $\sigma_{-}(\lambda)$, which is the sum of the 2 eigenvalues of $\mathbf{A}_{\infty}(\lambda)$ with non-negative real part, i.e., $\sigma_{-}(\lambda)=\mu_{m}^{-}(\lambda)+\mu_{p}^{-}(\lambda)$, and $\sigma_{-}(\lambda)$ is simple, an analytic function of $\lambda$, and has real part strictly greater than any other eigenvalue of $\mathbf{A}_{\infty}^{(2)}(\lambda)$. Note that $\sigma_{-}(\lambda)=-\sigma_{+}(\lambda)$ in this example.

Let $\zeta^{ \pm}(\lambda)$ be the eigenvectors associated with $\sigma_{ \pm}(\lambda)$, defined by

$$
\mathbf{A}_{\infty}^{(2)}(\lambda) \zeta^{+}(\lambda)=\sigma_{+}(\lambda) \zeta^{+}(\lambda) \quad \text { and } \quad \mathbf{A}_{\infty}^{(2)}(\lambda) \zeta^{-}(\lambda)=\sigma_{-}(\lambda) \zeta^{-}(\lambda) .
$$

These vectors can always be constructed in an analytic way (see [10]). From section 2.3] it follows that

$$
\zeta^{ \pm}(\lambda)=\operatorname{vec}_{m}^{ \pm}(\lambda) \wedge \operatorname{vec}_{p}^{ \pm}(\lambda) .
$$

This implies

$$
\zeta^{ \pm}=\left(F_{p}^{ \pm}+F_{m}^{ \pm},-2 e^{y} F_{p}^{ \pm} F_{m}^{ \pm}, \operatorname{sgn}(\Omega) \mathrm{i}\left(F_{m}^{ \pm}-F_{p}^{ \pm}\right), \operatorname{sgn}(\Omega) \mathrm{i}\left(F_{m}^{ \pm}-F_{p}^{ \pm}\right),-2 e^{-y}, F_{p}^{ \pm}+F_{m}^{ \pm}\right)^{T},
$$

The solution $\mathbf{U}^{ \pm}(x, \lambda)$ is the solution of the linearised system (2.11) with the property that $\lim _{x \rightarrow \pm \infty} e^{-\sigma_{ \pm}(\lambda) x} \mathbf{U}^{ \pm}(x, \lambda)=\zeta^{ \pm}(\lambda)$. In this example, dimension of the unstable manifolds at $x=\infty$ and $x=-\infty$ are the same. For the construction of the general case see [3, 10].

Note that the solutions $\mathbf{U}^{ \pm}(x, \lambda)$ are analytic expressions which represent the space of solutions which decay as $x \rightarrow \pm \infty$ in the original system (2.6). With this the Evans function can be defined as

$$
E(\lambda)=\mathrm{e}^{-\int_{0}^{x} \tau(s, \lambda) d s} \mathbf{U}^{-}(x, \lambda) \wedge \mathbf{U}^{+}(x, \lambda), \quad \lambda \in \Lambda,
$$

where $\wedge$ is the wedge product and

$$
\tau(x, \lambda)=\operatorname{Tr}(\mathbf{A}(x, \lambda)) .
$$

For the case of the coupled mode equation (1.2) this expression simplifies, since $\operatorname{Tr}(\mathbf{A}(x, \lambda))=$ 0, see (2.7). The Evans function, as defined above, can be extended across the continuous spectrum with some cuts in the complex plane, see GARDNER \& ZUMbrun 13] and Kapitula \& SANDSTEDE [14.

Next we will give an equivalent description of the Evans function, using the adjoint system. The adjoint system of (2.11) is

$$
\mathbf{V}_{x}=-{\overline{\left[\mathbf{A}^{(2)}(x)\right]}}^{T} \mathbf{V} \text {. }
$$

The dimension of the unstable manifold of this adjoint system is equal to the dimension of the stable manifold of the linearised system (2.11) and its most unstable eigenvalue is $-\overline{\sigma^{+}}$. Using 
the Hodge-star operator, we can relate the most unstable solution $\mathbf{V}^{-}$at $x=-\infty$ of the adjoint system with the most unstable solution $\mathbf{U}^{-}$of the linearised system at $x=-\infty$. Details can be found in [3, 6, 10]. To formulate the alternative description of the Evans function, we have to construct an inner product on $\bigwedge^{2}\left(\mathbb{C}^{4}\right)$. Let

$$
\mathbf{U}=\mathbf{u}_{1} \wedge \mathbf{u}_{2} \quad \text { and } \quad \mathbf{V}=\mathbf{v}_{1} \wedge \mathbf{v}_{2}, \quad \mathbf{u}_{i}, \mathbf{v}_{j} \in \mathbb{C}^{4}, \quad \forall i, j=1,2,
$$

be any decomposable 2 -forms. The inner product of $\mathbf{U}$ and $\mathbf{V}$ is defined by

$$
\llbracket \mathbf{U}, \mathbf{V} \rrbracket_{2}:=\operatorname{det}\left[\begin{array}{cc}
\left\langle\mathbf{u}_{1}, \mathbf{v}_{1}\right\rangle & \left\langle\mathbf{u}_{1}, \mathbf{v}_{2}\right\rangle \\
\left\langle\mathbf{u}_{2}, \mathbf{v}_{1}\right\rangle & \left\langle\mathbf{u}_{2}, \mathbf{v}_{2}\right\rangle
\end{array}\right]
$$

where $\langle\cdot, \cdot\rangle_{4}$ is the complex inner product in $\mathbb{C}^{4}$. Since every element in $\bigwedge^{2}\left(\mathbb{C}^{4}\right)$ is a sum of decomposable elements, this definition extends by linearity to any 2 -form in $\bigwedge^{2}\left(\mathbb{C}^{4}\right)$. An equivalent definition of the Evans function (2.14) is given by the following readily computable expression

$$
E(\lambda)=\llbracket \mathbf{V}^{-}(0, \lambda), \mathbf{U}^{+}(0, \lambda) \rrbracket_{2},
$$

where $\mathbf{V}^{-}(x, \lambda)$ is the most unstable solution at $x=-\infty$ of the adjoint system (2.16). From Section 2.3. it follows that the eigenvector of the adjoint matrix $-{\overline{\left(\mathbf{A}^{(2)}\right)}}^{T}$ for the eigenvalue $-\overline{\sigma_{+}}=\overline{\mu_{p}^{+}+\mu_{m}^{+}}$is

$$
\eta^{-}=\left(\overline{F_{p}^{+}+F_{m}^{+}},-2 e^{y} \overline{F_{p}^{+} F_{m}^{+}}, \operatorname{sgn}(\Omega) \mathrm{i}\left(\overline{F_{m}^{+}-F_{p}^{+}}\right), \operatorname{sgn}(\Omega) \mathrm{i}\left(\overline{F_{m}^{+}-F_{p}^{+}}\right),-2 e^{-y}, \overline{F_{p}^{+}+F_{m}^{+}}\right)^{T} .
$$

Hence the solution $\mathbf{V}^{-}(x, \lambda)$ is the solution of the adjoint linearised system (2.16) with the asymptotic behaviour $\lim _{x \rightarrow-\infty} e^{\overline{\sigma_{+}(\lambda)} x} \mathbf{V}^{-}(x, \lambda)=\eta^{-}(\lambda)$. The generalisation of this definition for general splittings and more details can be found in [6, 10].

For the numerical implementation, we will need a basis for $\bigwedge^{2}\left(\mathbb{C}^{4}\right)$, and the above construction assures that any basis will do. Therefore there is no loss of generality in assuming that the bases chosen are the standard ones. Starting with the standard basis for $\mathbb{C}^{4}$, and volume form $\mathcal{V}=\mathbf{e}_{1} \wedge \cdots \wedge \mathbf{e}_{4}$, let $\mathbf{a}_{1}, \ldots, \mathbf{a}_{6}$ be the induced orthonormal basis on $\bigwedge^{2}\left(\mathbb{C}^{4}\right)$. Using a standard lexical ordering, this basis can be taken to be

$$
\begin{aligned}
& \mathbf{a}_{1}=\mathbf{e}_{1} \wedge \mathbf{e}_{2}, \quad \mathbf{a}_{2}=\mathbf{e}_{1} \wedge \mathbf{e}_{3}, \quad \mathbf{a}_{3}=\mathbf{e}_{1} \wedge \mathbf{e}_{4}, \\
& \mathbf{a}_{4}=\mathbf{e}_{2} \wedge \mathbf{e}_{3}, \quad \mathbf{a}_{5}=\mathbf{e}_{2} \wedge \mathbf{e}_{4}, \quad \mathbf{a}_{6}=\mathbf{e}_{3} \wedge \mathbf{e}_{4} .
\end{aligned}
$$

Any $\mathbf{U} \in \bigwedge^{2}\left(\mathbb{C}^{4}\right)$ can be expressed as $\mathbf{U}=\sum_{j=1}^{6} U_{j} \mathbf{a}_{j}$. Since the basis elements $\mathbf{a}_{i}$ are orthogonal and the inner product $\llbracket \cdot, \cdot \rrbracket_{2}$ on $\bigwedge^{2}\left(\mathbb{C}^{4}\right)$ is equivalent to the inner product $\langle\cdot, \cdot\rangle_{6}$ on $\mathbb{C}^{6}$, the expression (2.17) for the Evans function can be expressed in the equivalent form

$$
E(\lambda)=\left\langle\mathbf{V}^{-}(0, \lambda), \mathbf{U}^{+}(0, \lambda)\right\rangle_{6}
$$

It is this form of the Evans function we implement in our numerical algorithm.

The matrix $\mathbf{A}^{(2)}: \bigwedge^{2}\left(\mathbb{C}^{4}\right) \rightarrow \bigwedge^{2}\left(\mathbb{C}^{4}\right)$ can be associated with a complex $6 \times 6$ matrix with entries

$$
\left\{\mathbf{A}^{(2)}\right\}_{i, j}=\llbracket \mathbf{a}_{i}, \mathbf{A}^{(2)} \mathbf{a}_{j} \rrbracket_{2}, \quad i, j=1, \ldots, 6,
$$


where, for any decomposable $\mathbf{U}=\mathbf{u}_{1} \wedge \mathbf{u}_{2} \in \bigwedge^{2}\left(\mathbb{C}^{4}\right), \mathbf{A}^{(2)} \mathbf{U}:=\mathbf{A} \mathbf{u}_{1} \wedge \mathbf{u}_{2}+\mathbf{u}_{1} \wedge \mathbf{A} \mathbf{u}_{2}$. Let $\mathbf{A}$ be an arbitrary $4 \times 4$ matrix with complex entries $a_{i j}, i, j=1, \cdots, 4$, then, with respect to the basis (2.18), $\mathbf{A}^{(2)}$ takes the explicit form

$$
\mathbf{A}^{(2)}=\left[\begin{array}{cccccc}
a_{11}+a_{22} & a_{23} & a_{24} & -a_{13} & -a_{14} & 0 \\
a_{32} & a_{11}+a_{33} & a_{34} & a_{12} & 0 & -a_{14} \\
a_{42} & a_{43} & a_{11}+a_{44} & 0 & a_{12} & a_{13} \\
-a_{31} & a_{21} & 0 & a_{22}+a_{33} & a_{34} & -a_{24} \\
-a_{41} & 0 & a_{21} & a_{43} & a_{22}+a_{44} & a_{23} \\
0 & -a_{41} & a_{31} & -a_{42} & a_{32} & a_{33}+a_{44}
\end{array}\right]
$$

Details for these constructions in more general systems can be found in [3].

\subsection{Integration scheme}

The subtle nature of the oscillatory instability requires a high order integration scheme for the numerical integration of the linearised and the adjoint system. Instead of using the second order Gauss-Legendre Runge-Kutta method, i.e. the implicit midpoint rule, as in BRIDGES, DeRKs \& GotTwald 10, we employ here a fourth order Gauss-Legendre scheme. We solve in $\mathbb{C}^{6}$

$$
\mathbf{U}^{n+1}=\mathbf{U}^{n}+\frac{1}{2} \Delta x\left(\mathbf{K}_{\mathbf{1}}+\mathbf{K}_{\mathbf{2}}\right),
$$

where $\Delta x$ is the spatial step size, sub- and superscripts $n$ denote the spatial discretization and $\mathbf{K}_{\mathbf{1 , 2}}$ are implicitly defined by

$$
\begin{aligned}
& \mathbf{K}_{\mathbf{1}}=\mathbf{A}^{(2)}\left(x_{n}+\left(\frac{1}{2}+\frac{\sqrt{3}}{6}\right) \Delta x\right) \times\left(\mathbf{U}^{n}+\frac{1}{4} \Delta x \mathbf{K}_{\mathbf{1}}+\left(\frac{1}{4}+\frac{\sqrt{3}}{6}\right) \Delta x \mathbf{K}_{\mathbf{2}}\right) \\
& \mathbf{K}_{\mathbf{2}}=\mathbf{A}^{(2)}\left(x_{n}+\left(\frac{1}{2}-\frac{\sqrt{3}}{6}\right) \Delta x\right) \times\left(\mathbf{U}^{n}+\frac{1}{4} \Delta x \mathbf{K}_{\mathbf{2}}+\left(\frac{1}{4}-\frac{\sqrt{3}}{6}\right) \Delta x \mathbf{K}_{\mathbf{1}}\right) .
\end{aligned}
$$

In practice we solve (2.22) for $\mathbf{K}_{\mathbf{1 , 2}}$ and then subsequently we can solve (2.21) for $\mathbf{U}^{\mathbf{n}+\mathbf{1}}$. Since all equations are linear, the implicit form of (2.22) can be cast in an explicit form.

The procedure for the numerical calculations is as follows. As explained in Section 2.4 it is sufficient to restrict the shooting algorithm to $\bigwedge^{2}\left(\mathbb{C}^{4}\right)$. As a starting vector for the shooting algorithm we use the eigenvectors of $\mathbf{A}_{\infty}^{(2)}(\lambda)$ in the far-field (see Sections 2.3 and 2.4). For the integration of the linearised system starting at $x=+L_{\infty}$ (with $L_{\infty} \gg 1$ ) the starting vectors for each $\lambda$ are the eigenvectors $\zeta^{+}(\lambda)$ related to the eigenvalues with the largest negative real part; for the integration of the adjoint system starting at $x=-L_{\infty}$ the starting vectors are the eigenvectors $\eta^{-}(\lambda)$ related to the eigenvalues with the largest positive real part. We have build in an analytical normalisation process such that the eigenvectors are normalized so that $\left\langle\eta^{-}(\lambda), \zeta^{+}(\lambda)\right\rangle_{6}=1$, for large values of $\lambda$. (Note that this normalisation is not used for values of $\lambda<2$.)

To calculate the Evans function, the linearised equation on $\bigwedge^{2}\left(\mathbb{C}^{4}\right)$

$$
\frac{d}{d x} \widetilde{\mathbf{U}}^{+}=\left[\mathbf{A}^{(2)}(x, \lambda)-\sigma_{+}(\lambda) \mathbf{I}_{d}\right] \widetilde{\mathbf{U}}^{+},\left.\quad \widetilde{\mathbf{U}}^{+}(x, \lambda)\right|_{x=L_{\infty}}=\zeta^{+}(\lambda),
$$

is integrated from $x=L_{\infty}$ to $x=0$, where the scaling

$$
\widetilde{\mathbf{U}}^{+}(x, \lambda)=e^{-\sigma_{+}(\lambda) x} \mathbf{U}^{+}(x, \lambda)
$$


ensures that any numerical errors due to the exponential growth are removed and $\left.\widetilde{\mathbf{U}}^{+}(x, \lambda)\right|_{x=0}=$ $\left.\mathbf{U}^{+}(x, \lambda)\right|_{x=0}$ is bounded. An alternative to this scaling is to impose a renormalization of the vectors during or at the end of the integration, with for example $\left|\widetilde{\mathbf{U}}^{+}(0, \lambda)\right|=1$, but such a scaling does not preserve analyticity.

For $x<0$, the adjoint equation

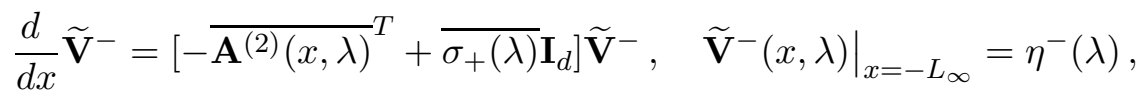

is integrated from $x=-L_{\infty}$ to $x=0$, also using the implicit midpoint rule, where again we introduce a rescaling

$$
\tilde{\mathbf{V}}^{-}(x, \lambda)=e^{\overline{\sigma_{+}(\lambda)} x} \mathbf{V}^{-}(x, \lambda)
$$

to remove the exponential growth.

At $x=0$, the computed Evans function is

$$
E(\lambda)=\left\langle\mathbf{V}^{-}(0, \lambda), \mathbf{U}^{+}(0, \lambda)\right\rangle_{6}=\left\langle\widetilde{\mathbf{V}}^{-}(0, \lambda), \widetilde{\mathbf{U}}^{+}(0, \lambda)\right\rangle_{6} .
$$

\section{Numerical results and discussion}

In this Section we show results of our algorithm for the detection of oscillatory instabilities in the perturbed massive Thirring model (1.2). We do not attempt here to present a thorough numerical analysis of the bifurcation scenarios of (1.2). The reader is referred to [5]. Instead our objective here is the presentation of a numerical algorithm which uses the Evans function as a numerical diagnostic tool for analysing edge bifurcations. Therefore we limit ourselves to illustrating several features of our numerical method. In Figure 1, we have sketched a cartoon which indicates where in parameter space, i.e., in the $\theta-\rho$-plane, the numerical analysis takes place. This cartoon is a guidance to help to locate the upcoming plethora of figures and how these figures are related to bifurcations and instabilities.

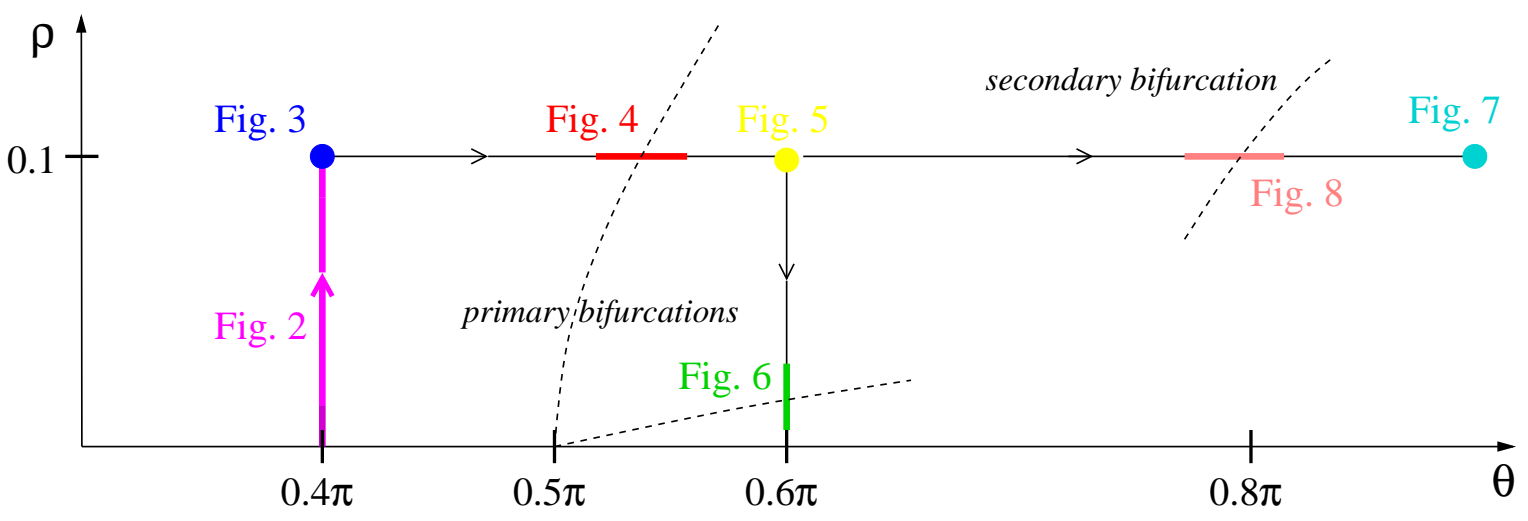

Figure 1: Cartoon to illustrate where in parameter space the numerical analysis takes place.

We study instability by computing the Evans function $E(\lambda)$ as defined in (2.19). The analyticity of the Evans function for $\Re(\lambda)>0$ allows one to detect oscillatory instabilities, i.e. complex roots of the Evans function, by means of Cauchy's theorem. The winding number of a closed curve in the $\lambda$-plane tells us about the number of unstable eigenvalues. In all our calculations, we compute the complex Evans function $E(\lambda)$, while varying the spectral parameter $\lambda=i \widetilde{\lambda}$ on the imaginary axis or varying the spectral parameter parallel to the imaginary axis with a (small) offset, explicitly $\lambda=$ off $+\mathrm{i} \widetilde{\lambda}$, where "off" is the offset. The normalisation of the Evans function is 
chosen such that the Evans function converges to 1 for $\lambda$ large. Hence the closed curve is formed by connecting the endpoints of the imaginary axis via the half-circle with infinite radius. On this half-circle, the Evans function will always have the value 1.

Since the system (1.2) has translational and rotational symmetry, the Evans function will have a fourth order zero at $\lambda=0$. This means that in the vicinity of $\lambda=0$, the Evans function scales as $E(\lambda) \sim \lambda^{4}$. Hence even an offset of only $10^{-4}$ yields that $E(\lambda)$ is of the order of $10^{-16}$, making the calculations meaningless. In order to avoid this problem, we use an offset of at least $5 \cdot 10^{-3}$ to analyse the Evans function near $\lambda=0$. Since we are interested here in oscillatory instabilities which occur at the edges of or within the continuous spectrum, and not in translational instabilities where eigenvalues emanate from $\lambda=0$, the offset near the $\lambda=0$ does not affect the detection of instabilities.
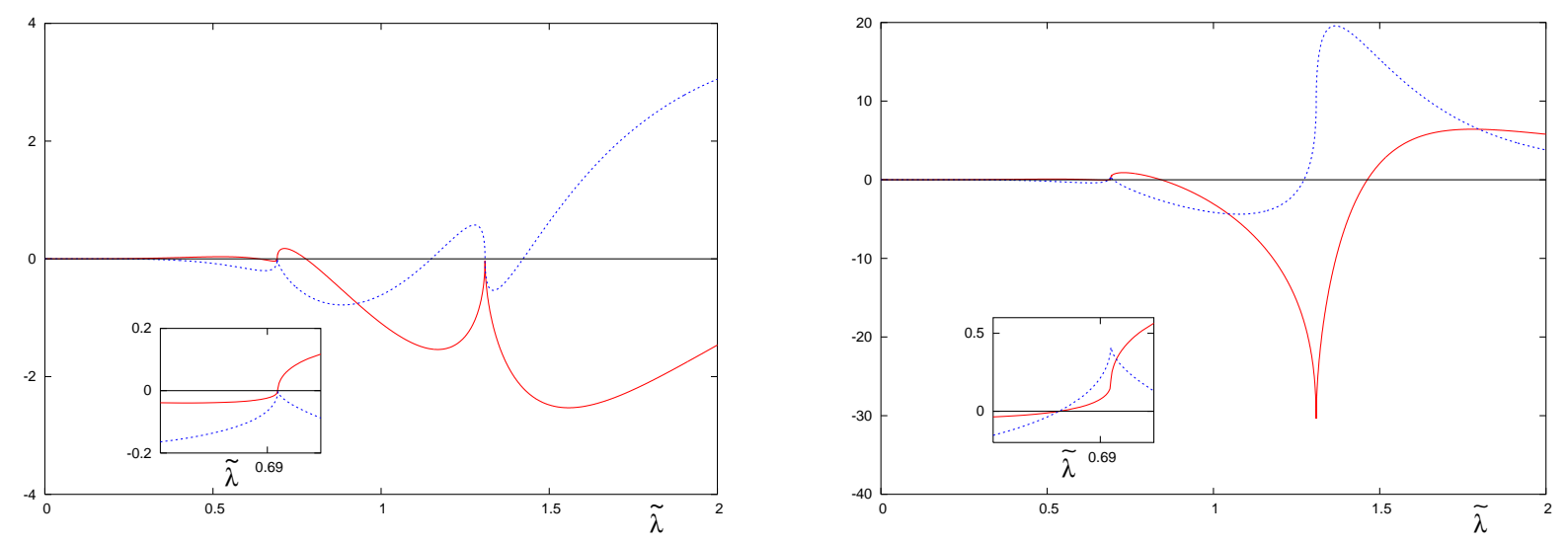

Figure 2: Real (continuous red line) and imaginary (dashed blue line) part of the Evans function $E(\lambda)$ as function of the spectral parameter $\lambda$ with off $=0$, for $V=0.9$ and $\theta=0.4 \pi$

(a) The integrable case $\rho=0$, The inset is a blow-up of the Evans function near the edge of the lower branch $\left(\lambda_{-}\right)$of the continuous spectrum.

(b): $\rho=0.1$. The inset is a blow-up of the Evans function and shows the discrete eigenvalue where $E(\lambda)=0$. The endpoints of the continuous spectrum occur at the cusps of the Evans function.

In the integrable case $(\rho=0)$, the linearised massive Thirring model (1.2) has only neutral and continuous eigenvalues [16, 17] and the solitary wave is stable for any $0<\theta<\pi$. The Evans function has zeros at the end points of the branches of continuous spectrum if $\rho=0$. In Figure 2 2 , we used our algorithm to calculate the Evans function for $\rho=0$, while the spectral parameter $\lambda$ is on the imaginary axis $(\mathrm{off}=0)$. This illustrates the zeros of the Evans function at the end points of the branches of continuous spectrum. As follows from Section 2.3 the edges of the continuous spectrum are located at $\lambda_{+}=i(|\Omega|+1)$ (upper branch) and at $\lambda_{-}=i(1-|\Omega|$ ) (lower branch). At these endpoints, the real or the imaginary part of the Evans function exibits a cusp, as can be seen in Figure 21. These cusps illustrate the non-analyticity of the Evans function at those points. Note that the cusps and the associated non-analyticity of the Evans function at the endpoints of the continuous spectrum occur for all $\rho>0$ and all $0<\theta<\pi$, as can be seen from the following figures.

For $0<\theta<\pi / 2$ and $\rho>0$, the linearised perturbed massive Thirring model (1.2) has a discrete eigenvalue, which is located on the imaginary axis in the gap between the branches of continuous spectrum, see results in [4, 15. This eigenvalue has bifurcated at $\rho=0$ from the lower end point $\left(\lambda_{-}\right)$of the continuous spectrum. Our algorithm can follow this discrete eigenvalue on the imaginary axis, as illustrated in Figure $2 \mathrm{~b}$. The edges of the two continuous branches corresponding to positive and negative energy states in the massive Thirring model (1.2) are 
located on the imaginary axis at $\lambda_{+}=i\left(|\Omega|+1\right.$ ) (upper branch) and at $\lambda_{-}=i(1-|\Omega|$ ) (lower branch) (see Section [2.3). Here the discrete eigenvalue which lies on the imaginary axis is clearly below the edge of the lower branch $\left(\lambda_{-}\right)$of the continuous spectrum. In the gap between the branches of continuous spectrum, the Evans function is still analytic, since there is still a 2:2 split of the dimensions of the stable and unstable manifolds.

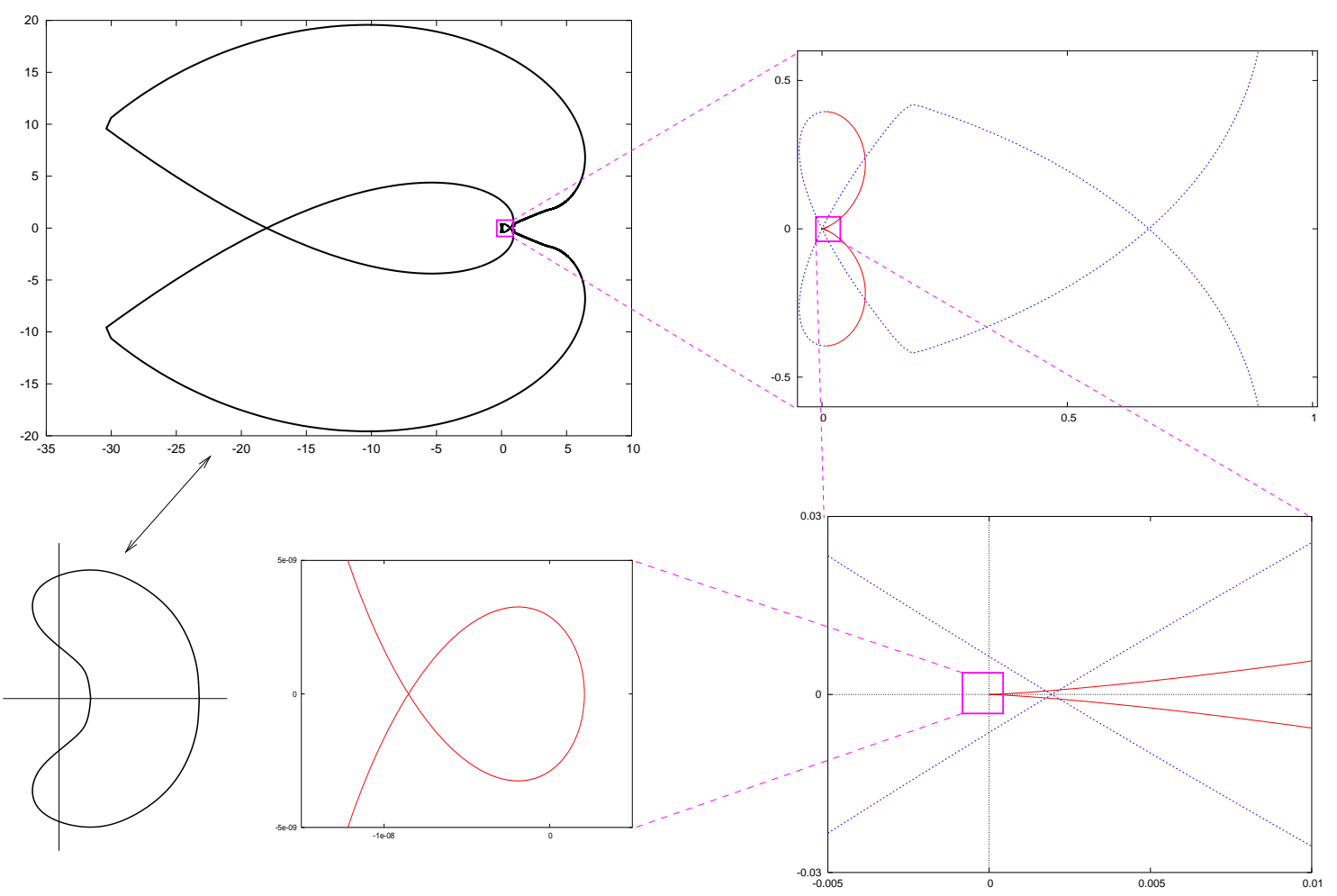

Figure 3: The real versus imaginary parts of the Evans function $E(\lambda)$ for the non-integrable case $\rho=0.1, V=0.9$ and $\theta=0.4 \pi$. The spectral parameter $\lambda$ varies parallel to the imaginary axis with a small offset off $=10^{-11}$. Going round clockwise from the upper left corner:

(a): Overview of the Evans function.

(b): First zoom into the area near $E=0$. The colours are used to help identifying the lines in the next picture.

(c): Next zoom into this area. This picture shows that the two little loops cross right of the zero point. For visualisation purposes, we used off $=5 \cdot 10^{-5}$. At off $=0$ the crossing of the loops is located exactly at the origin, indicating the discrete eigenvalue on the imaginary axis. The slight offset off $=5 \cdot 10^{-5}$ moves the crossing of the loops to the right of the origin.

(d): Final zoom into the area near $E=0$ (note how small the scale is). We have increased the offset to off $=5 \cdot 10^{-3}$ to avoid problems with the smallness of the Evans function near $\lambda=0$.

(e): A topologically equivalent sketch of the Evans function.

For $0<\theta<\pi / 2$, the solitary waves are known to be stable even for non-zero $\rho$ [4, 15. In Figure 3 we show the Evans function for such a stable case. To determine the winding number, we need to zoom into the neighbourhood of $E=0$. The behaviour near $E=0$ (Figure 3 bd) and the global behaviour of the Evans function (Figure 3a) convey that the Evans function is topologically equivalent to a loop which does not contain the origin (Figure 3.). Hence the winding number is zero, confirming stability,

For $\pi / 2<\theta<\pi$, instabilities may arise for non-zero $\rho$. These instabilities are of an oscillatory nature in the sense that discrete eigenvalues leave the imaginary axis into the complex plane [4, 15]. 

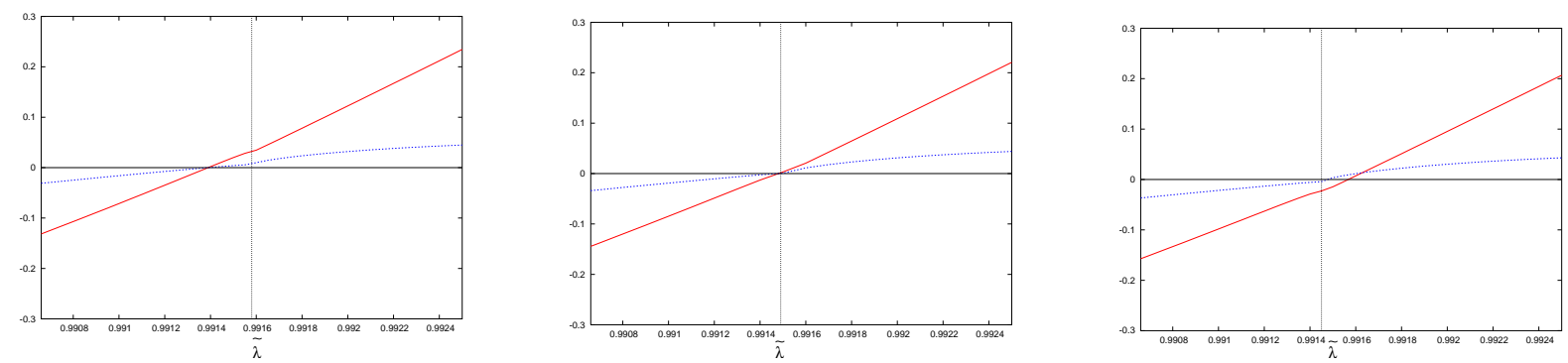

Figure 4: The onset of the primary instability for $\rho=0.1$ and $V=0.9$ (the offset off $=0$ ). Plotted are the real part (continuous red line) and imaginary part (dashed blue line) of $E(\lambda)$ versus $\lambda$. The vertical dotted line indicates the edge of the lower branch $\left(\lambda_{-}\right)$of the continuous spectrum. In the left picture with $\theta=0.50268 \pi$, the eigenvalue is still located on the imaginary axis. In the middle picture with $\theta=0.50270 \pi$, the eigenvalue has just merged with the end point of the lower branch $\left(\lambda_{-}\right)$of the continuous spectrum, giving a zero of the Evans function on the imaginary axis at the end of the continuous spectrum. In the right picture with $\theta=0.50272 \pi$, there is no eigenvalue on the imaginary axis anymore.

The onset of the first instability is when the discrete eigenvalue in the gap of the continuous spectrum merges with the edge of the lower branch $\left(\lambda_{-}\right)$of the continuous spectrum. In the $\theta-\rho$ bifurcation plane, the instability curve starts at $\rho=0$ and $\theta=0.5$ initially proportional to $\sqrt{\rho}$ 4, 15. In Figure 4 the onset of the instability is illustrated at $\rho=0.1$ and $V=0.9$. At
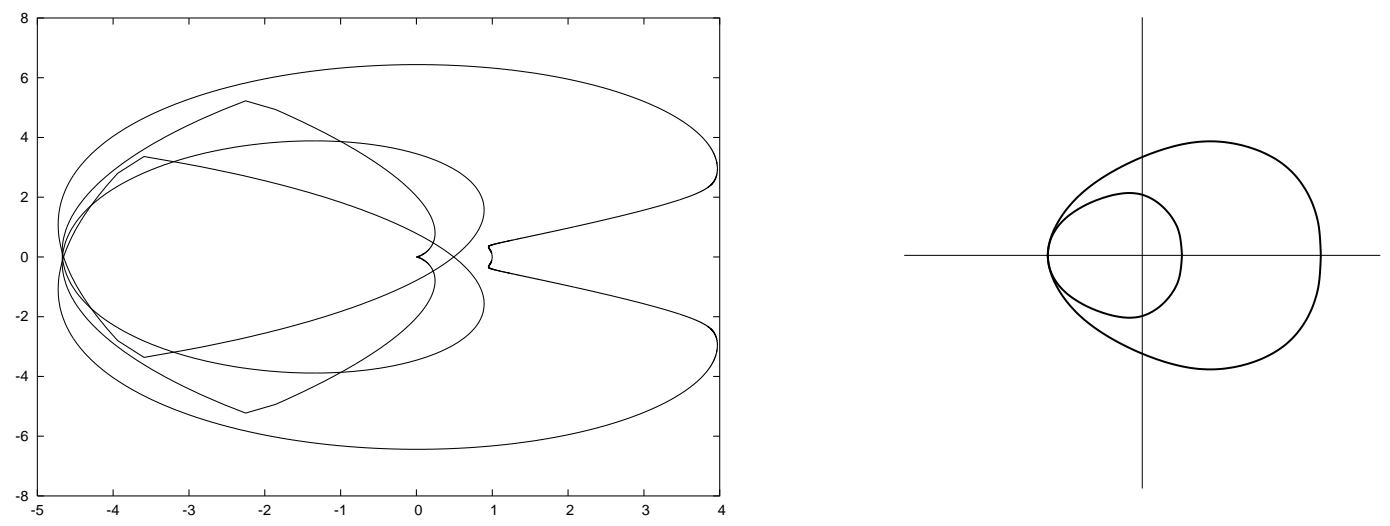

Figure 5: (a): The real versus imaginary parts of the Evans function $E(\lambda)$ for $\rho=0.1, V=0.9$ and $\theta=0.6 \pi$. The spectral parameter $\lambda$ varies parallel to the imaginary axis with off $=5 \cdot 10^{-14}$. (b): The Evans function in Figure [5a is topologically equivalent to this sketch of $E(\lambda)$. The winding number is clearly 2, confirming that there is one pair of unstable eigenvalues for these parameter values.

the bifurcation point the eigenvalue detaches from the imaginary axis at the edge of the lower branch $\left(\lambda_{-}\right)$of the continuous spectrum and leaves into the complex plane. This can be explored by looking at the winding number of the Evans function. Figure 5 shows the real and imaginary parts of the Evans function when the instability has well occurred (at $\rho=0.1, V=0.9$ and $\theta=0.6 \pi)$. The winding number is 2 , confirming that a pair of unstable eigenvalues is present.

If we now decrease $\rho$ (with $\theta$ fixed) from the above described primary bifurcation of oscillatory instability, the primary unstable eigenvalue will merge with the edge of the upper branch $\left(\lambda_{+}\right)$of the continuous spectrum. This is illustrated in Figure 6. If $\rho$ is decreased below this bifurcation, the solitary wave is stable again. We see clearly the collision of the eigenvalue with the edge 
of the upper branch $\left(\lambda_{+}\right)$of the continuous spectrum. In [15], a slightly different perturbation is studied and it is proved analytically that in this case two bifurcation curves originate from $(\theta, \rho)=(0.5 \pi, 0)$. However, it is not clear what the relation with the branches of continuous spectrum is away from the bifurcation point. Our calculations suggest that for our perturbation there are again two bifurcation curves, one curve is related to a bifurcation from the edge of the lower branch $\left(\lambda_{-}\right)$and the other curve to a bifurcation from the edge of the upper branch $\left(\lambda_{+}\right)$and the primary unstable eigenvalue goes between these two branches. This seems a new observation, which has not been recognised earlier.
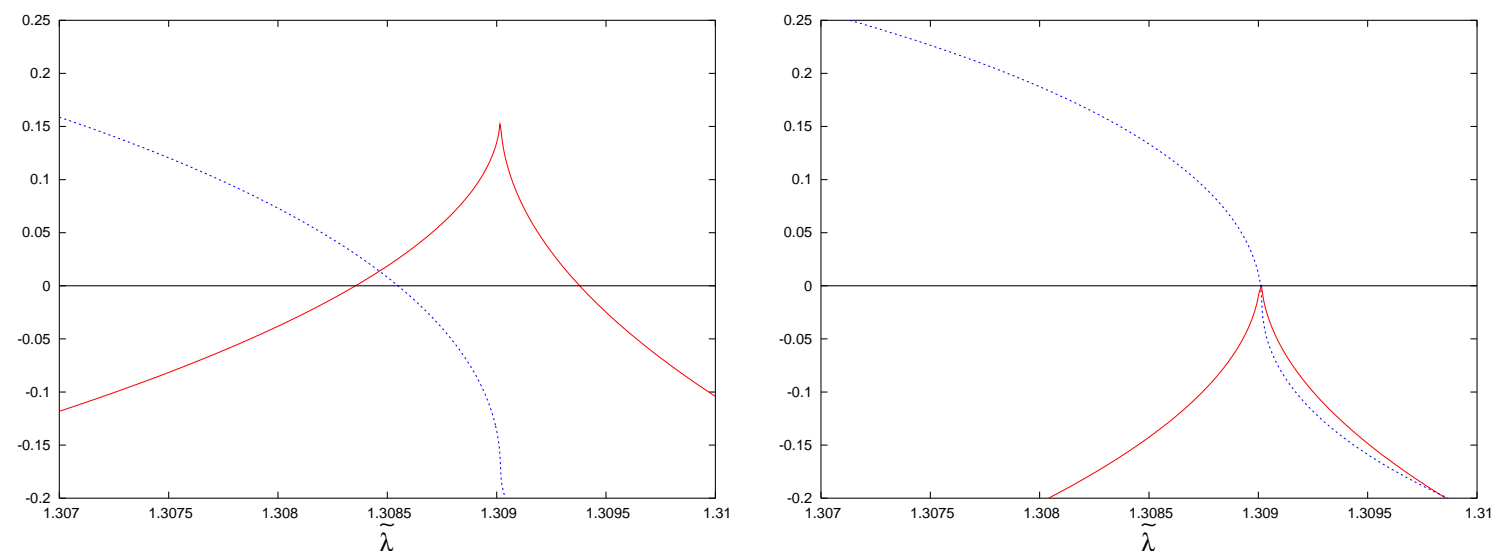

Figure 6: Real (continuous red line) and imaginary part (dashed blue line) of the Evans function $E(\lambda)$ as a function of the spectral parameter $\lambda$ for $V=0.9$ and $\theta=0.6 \pi$ (the offset off $=$ $\left.5 \cdot 10^{-14}\right)$. (a): Above the bifurcation point at $\rho=0.002$. (b): At the bifurcation point $\rho=0.0001$.

Figures 4 and 6illustrate that our algorithm and the Evans function allow us to exactly follow the eigenvalues in the course of the bifurcation. By looking at the real and imaginary part of the Evans function the value of lambda which corresponds to a root of the Evans function can be located. This allowed us to answer the question from where on the imaginary axis the eigenvalues detach for the primary instability.

It has been found analytically in [4] that secondary bifurcations can occur for suitable values of $\rho$ and $\theta$ (we assume $V$ fixed here for simplicity). Here a second pair of eigenvalues detach from the continuous spectrum into the complex plane. Again, analyticity of the Evans function allows us to compute the number of unstable eigenvalues by determing the winding number. In Figure 7 we show that the winding number equals 4 when $\rho=0.1, V=0.9$ and $\theta=0.9 \pi$, indicating that a new pair of eigenvalues have detached. The behaviour near $\lambda=0$ close to the origin of the Evans function behaves is similar to the one depicted in Figure $3 \mathrm{~d}$. The onset of this secondary instability at $\rho=0.1$ and $V=0.9$ is shown in Figure 8 Figure 8 shows the behaviour of the Evans function shortly before, at, and shortly after the secondary instability. In order to see the secondary instability and the emergence of the eigenvalue, one needs to look at the behaviour near $E=0$. Note that the secondary instability is hard to observe in the full Evans function (left pictures in Figure 8). In the middle picture, the Evans function has a zero, just after the edge of the upper branch $\left(\lambda_{+}\right)$of the continuous spectrum, but not at the edge of this branch. Hence for $\rho>0$, the eigenvalue comes really from within the continuous spectrum, not out of one the endpoints of the branches of the continuous spectrum. Similar behaviour has been described by SANDSTEDE \& Scheel [23].

The results in this section show that our algorithm using the Evans function approach exercised on the wedge space provides a good and reliable diagnostic tool for the accurate detection 

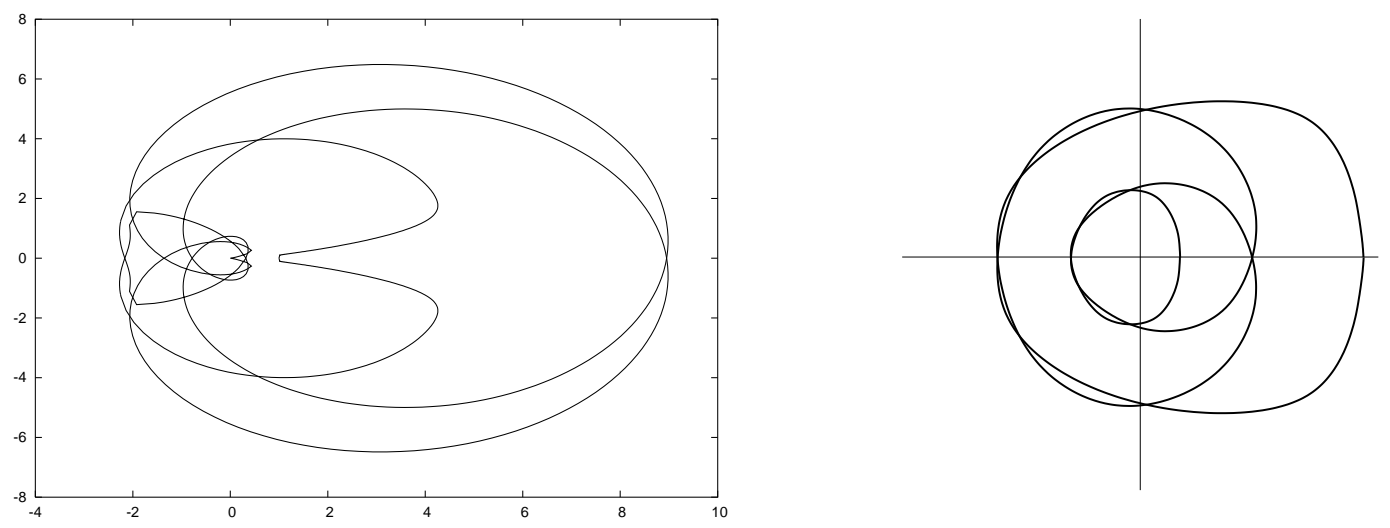

Figure 7: (a): The real versus imaginary parts of the Evans function $E(\lambda)$ for $\rho=0.1, V=0.9$ and $\theta=0.9 \pi$. The spectral parameter $\lambda$ varies parallel to the imaginary axis with off $=5 \cdot 10^{-14}$. (b): The Evans function in (a) is topologically equivalent to this picture. The winding number is clearly 4, confirming that there are two pairs of unstable eigenvalues for these parameter values.

of oscillatory instabilities. The analyticity of the Evans function allows for detection of complex eigenvalues by assuring the necessary conditions for the application of Cauchy's principle. Our algorithm is able to follow the location of the discrete eigenvalues in the course of their bifurcations. The Evans function does not involve a discretization of the spectrum of the eigenvalue problem and hence does not fracture the continuous spectrum. It is therefore free of spurious unstable eigenvalues. Moreover, the exterior algebra is a platform for numerically stable shooting. As already stated, the method is not restricted to the particular coupled mode model (1.2) but can be applied to other problems as well.

\section{Acknowledgements}

We would like to thank Thomas Bridges and Dmitry Pelinovsky for valuable discussions. Gianne Derks' research was partially supported by a European Commission Grant, contract number HPRN-CT-2000-00113, for the Research Training Network Mechanics and Symmetry in Europe (MASIE), http://www.ma.umist.ac.uk/jm/MASIE/

\section{References}

[1] A. B. Aceves \& S. Wabnitz. Self-induced transparency solitons in nonlinear refractive periodic media. Phys. Lett. A141, 37-42 (1989).

[2] J. Alexander, R. Gardner \& C.K.R.T. Jones. A topological invariant arising in the stability analysis of traveling waves, J. Reine Angew. Math. 410, 167-212 (1990).

[3] L. Allen \& T.J. BRIDges. Numerical exterior algebra and the compound matrix method, Numerische Mathematik 92, 197-232 (2002)

[4] I.V. Barashenkov, D.E. Pelinovsky \& E.V. Zemlyanaya. Vibrations and oscillatory instabilities of gap solitons, Phys. Rev. Lett. 80, 5117-5120 (1998).

[5] I.V. Barashenkov \& E.V. Zemlyanaya. Oscillatory instabilities of gap solitons: a numerical study, Comp. Phys. Comm. 126, 22-27 (2000).

[6] S. Benzoni-Gavage, D. Serre \& K. Zumbrun. Alternate Evans functions and viscous shock waves, SIAM J. Math. Anal. 32, 929-962 (2001). 
[7] L.Q. Brin. Numerical testing of the stability of viscous shock waves, Math. Comp. 70, 10711088 (2001).

[8] L.Q. Brin \& K. Zumbrun. Analytically varying eigenvectors and the stability of viscous shock waves, Preprint, Indiana University (2002).

[9] D. K. Campbell \& A. R. Bishop. Soliton excitations in polyacetylene and relativistic field-theory models, Nucl. Phys. B200, 297-328 (1982).

[10] T. Bridges, G. Derks \& G. A. Gotwwald. Stability and instability of solitary waves of the fifth-order KdV equation: a numerical framework, Physica D172, 190-216 (2003).

[11] C. M. De Sterke \& J. E. Snipe. In Progress in Optics, Vol. XXXIII, E. Wolf (Ed.), Elsevier Science, Amsterdam, 1994), p.203.

[12] J.W. Evans. Nerve axon equations IV. The stable and unstable impulse, Indiana Univ. Math. J. 24, 1169-1190 (1975).

[13] R. Gardner and K. Zumbrun The gap lemma and geometric criteria for instability of viscous shock profiles, Comm. Pure Appl. Math. 51, 797-855 (1998).

[14] T. Kapitula \& B. Sandstede. Stability of bright solitary wave solutions to perturbed nonlinear Schrödinger equations, Phys. D 124, 58-103 (1998).

[15] T. Kapitula \& B. SAndstede. Edge bifurcations for near integrable systems via Evans function techniques, SIAM J. Math. Anal. 33, 1117-1143 (2002).

[16] D. J. Kaup \& T. I. Lakoba. The squared eigenfunctions of the massive Thirring model in laboratory coordinates, J. Math. Phys. 37, 308-323 (1996)

[17] D. J. Kaup \& T. I. Lakoba. Variational method: How it can generate false instabilities, J. Math. Phys. 37, 3442-3462 (1996)

[18] Yu. S. Kivshar, O. A. Chubyakalo, O. V. Ustatenko \& D. V. Grinyoff. Bright and dark gap solitons governed by quadratic nonlinearities, Int. J. Mod. Phys. 9, 2963-2987 (1995).

[19] S. Y. LeE, T. K. KuO \& A. GAVRIELIDES. Exact localized solutions of two-dimensional field theories and massive fermions with Fermi interaction, Phys. Rev. D12, 2249-2253 (1975).

[20] R. Friedberg \& T. D. LeE. Fermion-field nontopological solitons, Phys. Rev. D15, 16941711 (1977).

[21] M. Marcus. Finite Dimensional Multilinear Algebra, Part II, Marcel Dekker: New York (1975).

[22] M. Romangoli, S. Trillo \& S. Wabnitz. Soliton switching in nonlinear couplers, Opt. Quantum. Electron. 24, S1237-S1267 (1992).

[23] B. Sandstede And A. Scheel. Absolute and convective instabilities of waves on unbounded and large bounded domains, Physica D 145, 233-277 (2000).

[24] B. Sandstede And A. Scheel. On the stability of travelling waves with large spatial period, J. Diff. Eq. 172, 134-188 (2001). 

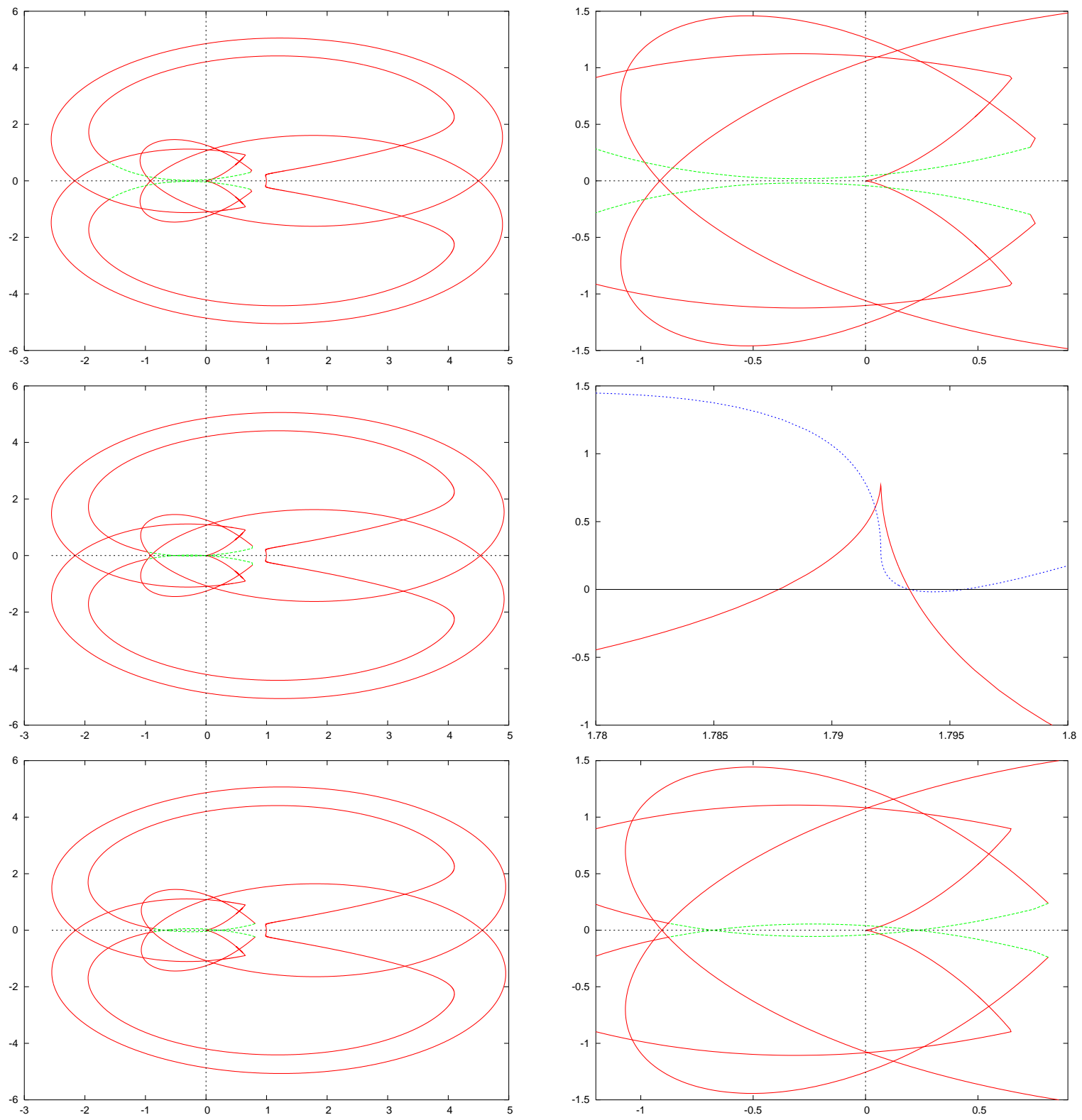

Figure 8: The real versus imaginary parts of the Evans function $E(\lambda)$ for $\rho=0.1, V=0.9$ and $\theta$ varying. The spectral parameter $\lambda$ varies on the imaginary axis (off $=0$ ). The green line helps to locate the emergence of the second eigenvalue.

(a): Just before the onset of the secondary instability at $\theta=0.790$. The right picture zooms in at the neighbourhood of $E=0$.

(b): At the onset of instability at $\theta=0.791$. The right picture shows the real (continuous red line) and imaginary (dashed blue line) parts of the Evans function as function of $\lambda$. This shows clearly that the zero of the Evans function occurs just after the edge of the upper branch $\left(\lambda_{+}\right)$of continuous spectrum (i.e., the cusp), hence inside the continuous spectrum and not at the edge.

(c): Just after the onset of the secondary instability at $\theta=0.792$. The right picture zooms into the neighbourhood of $E=0$. The loops with the green line now include the origin $(E=0)$, increasing the winding number by 2 and illustrating that an additional instability has occurred. 